\title{
Treatment and Outcomes of Metastatic Pancreatic Cancer in Elderly Patients
}

\author{
Hyun Ae Junga, b Bo Ram Han ${ }^{c}$ Ho Young Kim ${ }^{c}$ Hyo Jung Kim ${ }^{c}$ \\ Dae Young Zang ${ }^{c}$ Joo Young Jung ${ }^{b}$

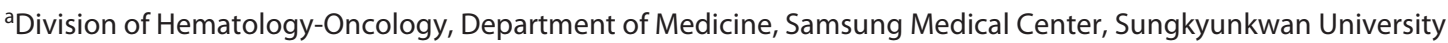 \\ School of Medicine, Seoul, South Korea; bepartment of Internal Medicine, Hallym University Medical Center, \\ Hallym University College of Medicine, Hwaseong-si, South Korea; 'Department of Internal Medicine, Hallym \\ University Medical Center, Hallym University College of Medicine, Anyang, South Korea
}

\section{Keywords}

Chemotherapy · Old age · Pancreatic cancer · Palliative treatment

\begin{abstract}
Background and Aims: Although pancreatic cancers are common in older age-groups, the prognosis remains poor due to limited studies on treatment approaches and outcomes in a given population. We aimed to examine treatment patterns and their outcomes in older patients with metastatic pancreatic cancer in a real-world context. Materials and Methods: We conducted a retrospective study including 167 patients with metastatic pancreatic cancer (aged $\geq 70$ years and male/female: $78 / 89$ ) between January 2010 and July 2015. Patients' retrieved data from medical records were analyzed according to treatment types, followed by a review of clinicopathologic variables and treatment outcomes. Results: Of the 167 eligible patients for the study, only $21.6 \%(n=36)$ received palliative chemotherapy. The median age of the chemotherapy group was 74.0 years and 78.6 years for the supportive care group. The median survival of the chemotherapy group was 9.2 months (range: 1.0-24.9 months), compared with that of the sup-
\end{abstract}

portive care group, which was 2.3 months (range: 0.1-31.8 months). Among the patients in the chemotherapy group, $50 \%(n=18)$ received gemcitabine-based double therapy, and $30 \%$ patients $(n=9)$ received second-line chemotherapy. Conclusions: Our results showed that older patients with metastatic pancreatic cancer were less likely to receive chemotherapy. However, the survival benefit from chemotherapy was comparable to that of younger patients' counterpart. Thus, further study involving identification of older patients who would benefit from cytotoxic chemotherapy is needed.

(C) 2021 The Author(s)

Published by S. Karger AG, Basel

\section{Introduction}

The incidence of cancer in the older population is rising, owing to increasing life expectancy. Additionally, the number of older patients with cancer has increased and is expected to continuously increase with advances in cancer treatment [1]. Cancer typically develops in older people aged $\geq 55$ years. In the USA, for example, $80 \%$ of all cancers are diagnosed in people aged $\geq 55$ years, according to the American Cancer Society. 
Pancreatic cancer is considered one of the most aggressive forms of cancer in which $>75 \%$ of patients are diagnosed with locally advanced or metastatic disease, mainly affecting the older population with a median age of 71 years at diagnosis. Meanwhile, the incidence of pancreatic cancer has increased in recent years, and $53 \%$ of patients are diagnosed initially with metastatic disease already in existence. Despite this large pancreatic patients' population, the older individuals have often been excluded from clinical trials and active cancer treatment. A report has indicated that only $5 \%$ of older patients with metastatic pancreatic cancer were enrolled in the clinical trial [2]. Thus, the benefit of systemic chemotherapy for elderly patients in this condition in a palliative setting is not well-known. Also, the choice of chemotherapy regimens for an individual patient may be a common clinical situation, considering factors, such as the extent of disease, prognosis, activity of chemotherapeutic agents, and potential toxicity, especially for those who are $>70$ years or those who have decreased performance status. Unfortunately, due to the exclusion of older patients in clinical trials, clinicians are challenged by the choice of chemotherapy regimen for this group of individuals after new drug approval.

Gemcitabine was approved as a first-line agent based on a pivotal phase III clinical trial for advanced pancreatic cancer in 1996 [3]. Gemcitabine remains the mainstay of first-line treatment for pancreatic cancer in our setting. In a study of pancreatic cancer patients who were $>65$ years, only $54 \%$ received chemotherapy [4]. The introduction of combination chemotherapy regimen, such as gemcitabine plus nab-paclitaxel [5] and 5-fluorouracil/leucovorin with irinotecan and oxaliplatin (FOLFIRINOX) has significantly improved outcomes, compared with gemcitabine monotherapy. Furthermore, in a phase III randomized study that enrolled 861 metastatic pancreatic cancer patients to evaluate the efficacy and safety of nabpaclitaxel plus gemcitabine combination versus gemcitabine monotherapy, it was found that the median overall survival (OS) was 8.5 months in the former and 6.7 months in the latter group (hazard ratio: 0.72 and $p<$ $0.001)$. The median progression-free survival and the objective response rate were also improved in the combination arm, and toxicity related to the nab-paclitaxel addition was manageable. A combination chemotherapy regimen, especially FOLFIRINOX, is considered the first-line therapy for pancreatic cancer patients with good performance status [6], and the administration of the combined nab-paclitaxel plus FOLFIRINOX regimen for pancreatic cancer has generally been manageable [7-9]; nevertheless, it would be of interest to know the effectiveness of the regimen in older patients. To date, there is no consensus on patient management of chemotherapy regimens for the elderly, and often, either they do not receive therapy or they settle for suboptimal doses of chemotherapy due to the general assumption that older individuals cannot tolerate chemotherapy. Research on the identification of key prognostic factors for pancreatic cancer in older patients is warranted to permit optimal selection and stratification of patients who can best tolerate and benefit from systemic therapy. Although pancreatic cancers are common in older age-groups, treatment approaches and outcomes are less investigated in this population. We analyzed the data to compare treatment outcomes for older patients with metastatic pancreatic cancer retrospectively.

\section{Methods}

\section{Patients and Data Collection}

We conducted a retrospective study, enrolling 167 patients (aged $\geq 70$ years) with histologically confirmed metastatic or recurrent pancreatic adenocarcinoma from January 2010 to July 2015. All demographics and relevant clinicopathologic data were retrieved from the patients' medical records. Subsequently, the clinicopathologic variables and treatment outcomes were reviewed. This study was approved by the Institutional Review Board of Hallym Medical Center with a waiver for informed consent due to the retrospective study design. The ethical principles established by the Helsinki Declaration were followed.

\section{Statistical Analyses}

We performed survival analyses by estimating the OS (from the day of diagnosis to the day of death or the last follow-up). The OS was assessed using the Kaplan-Meier method, and the log-rank test was used to test the differences between OS associated with the clinical variables. Statistical significance of the factors associated with OS was investigated using the univariate and multivariate Cox proportional hazards regression models. Hazard ratios and their $95 \%$ confidence intervals were computed. $p$ value $<0.05$ was considered a statistically significant difference. All statistical analyses were performed using IBM SPSS Statistics 24 (Armonk, NY, USA).

The severity of comorbid diseases was recorded and scored according to the Charlson Comorbidity Index (CCI) [10]. Patients were divided into 3 groups as follows: Group 1: mild, with CCI scores of 0 ; Group 2: moderate, with CCI scores of 1; and Group 3: severe, with CCI scores $\geq 2$.

\section{Results}

\section{Patient Characteristics}

We recruited 167 patients aged $\geq 70$ years with metastatic pancreatic cancer, who were eligible for the study. Table 1 shows the baseline characteristics of the study co- 
Table 1. Patients' baseline demographic and clinical characteristics

\begin{tabular}{|c|c|c|}
\hline Characteristics & $n$ & $\%$ \\
\hline \multicolumn{3}{|l|}{$\operatorname{Sex}$} \\
\hline Male & 78 & 46.7 \\
\hline Female & 89 & 53.3 \\
\hline \multicolumn{3}{|l|}{ Age } \\
\hline$<80$ & 114 & 68.3 \\
\hline$\geq 80$ & 53 & 31.7 \\
\hline \multicolumn{3}{|l|}{ ECOG } \\
\hline $0-1$ & 81 & 48.5 \\
\hline 2 & 86 & 51.5 \\
\hline \multicolumn{3}{|l|}{ CCI } \\
\hline 0 & 51 & 30.5 \\
\hline 1 & 58 & 34.7 \\
\hline$\geq 2$ & 58 & 34.7 \\
\hline \multicolumn{3}{|l|}{ Site of primary } \\
\hline Head & 56 & 33.5 \\
\hline Body & 75 & 44.9 \\
\hline Tail & 36 & 21.6 \\
\hline \multicolumn{3}{|c|}{ Previous operation history } \\
\hline Yes & 35 & 21.0 \\
\hline No & 132 & 79.0 \\
\hline \multicolumn{3}{|l|}{ Metastasis } \\
\hline Lung & 43 & 25.1 \\
\hline Liver & 78 & 46.7 \\
\hline Peritoneum & 12 & 7.2 \\
\hline LN & 42 & 25.0 \\
\hline
\end{tabular}

ECOG, Eastern Cooperative Oncology Group; CCI, Charlson Comorbidity Index.

hort population. Of these, $21.6 \%$ patients received palliative chemotherapy. The median age was 74.0 years (range: $70-84$ years) in the chemotherapy group and 78.6 years (range: 70-94.6 years) in the supportive care group. Fiftythree $(31.7 \%)$ patients were $>80$ years old, and $78(46.7 \%)$ were male. The metastatic locations were comparable to those observed in pancreatic cancer, which includes the lung, liver, peritoneum, and lymph node. Thirty-five (21\%) patients had relapse of the disease after prior curative resection. Between supportive care and active chemotherapy groups, the scores for the Eastern Cooperative Oncology Group (ECOG) scale of performance status showed a significant difference, although the CCI was similar (Table 2). Among patients with good performance (ECOG $0-1)$ and low CCI (0-1), 36 of 58 patients received chemotherapy. Thirty-seven percent of good performance and low CCI decline chemotherapy due to concern of the side effect of chemotherapy.

Treatment for Elderly Patients with Metastatic Pancreatic Cancer
Table 2. Difference of ECOG and CCI between the supportive care and the chemotherapy groups

\begin{tabular}{llll}
\hline & \multicolumn{2}{l}{ Treatment } & $p$ value \\
\cline { 2 - 3 } & supportive care, \% & chemotherapy, \% & \\
\hline ECOG & & $34(94.4)$ & $<0.001$ \\
$0-1$ & $47(35.9)$ & $2(5.6)$ & \\
2 & $84(64.1)$ & $11(33.4)$ & 0.173 \\
$C C I$ & $58(44.3)$ & $20(60.6)$ & \\
0 & $61(46.6)$ & $2(6.1)$ & \\
1 & $12(9.1)$ & & \\
$\geq 2$ & & & \\
\hline
\end{tabular}

ECOG, Eastern Cooperative Oncology Group; CCI, Charlson Comorbidity Index.

Table 3. Univariate and multivariate analysis

\begin{tabular}{|c|c|c|c|c|}
\hline \multirow[t]{2}{*}{ Characteristics } & \multicolumn{2}{|l|}{ Univariate } & \multicolumn{2}{|c|}{$\begin{array}{l}\text { Multivariate } \\
\text { analysis }\end{array}$} \\
\hline & median OS & $p$ value & HR & $p$ value \\
\hline \multicolumn{5}{|l|}{$\operatorname{Sex}$} \\
\hline Male & 3.9 & 0.101 & & \\
\hline Female & 2.8 & & & \\
\hline \multicolumn{5}{|l|}{ Age } \\
\hline$<80$ & 4.57 & 0.004 & 1.29 & 0.168 \\
\hline$\geq 80$ & 2.33 & & & \\
\hline \multicolumn{5}{|l|}{$\overline{E C O G}$} \\
\hline $0-1$ & 9.17 & $<0.001$ & 5.67 & $<0.001$ \\
\hline 2 & 1.30 & & & \\
\hline \multicolumn{5}{|l|}{$C C I$} \\
\hline 0 & 6.46 & $<0.001$ & 1.08 & $<0.001$ \\
\hline 1 & 5.90 & & & \\
\hline$\geq 2$ & 0.97 & & & \\
\hline \multicolumn{5}{|l|}{ Site of primary } \\
\hline Head & 4.50 & 0.385 & & \\
\hline Body & 2.56 & & & \\
\hline Tail & 3.85 & & & \\
\hline \multicolumn{5}{|l|}{ Previous op history } \\
\hline Yes & 3.90 & 0.539 & & \\
\hline No & 2.00 & & & \\
\hline \multicolumn{5}{|l|}{ Treatment } \\
\hline Supportive care & 2.50 & $<0.001$ & 0.65 & 0.042 \\
\hline Palliative chemotherapy & 9.80 & & & \\
\hline
\end{tabular}

ECOG, Eastern Cooperative Oncology Group; CCI, Charlson Comorbidity Index; OS, overall survival; HR, hazard ratio. 


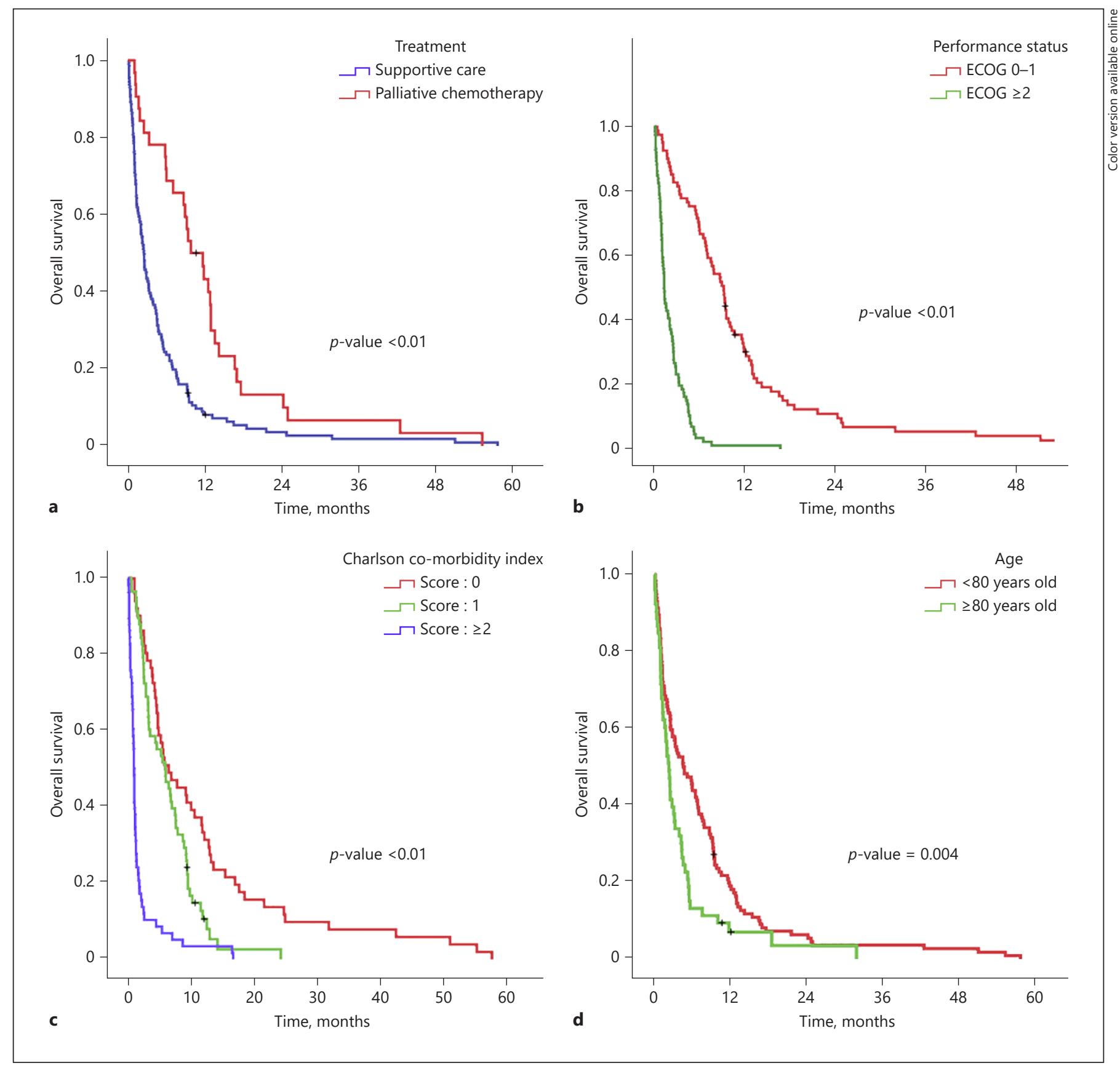

Fig. 1. a OS in the chemotherapy group vs supportive group. b OS by ECOG. c OS by Charlson Comorbidity Index.d OS by age. OS, overall survival.

\section{Survival}

The median survival was 9.2 months (range: 1.0-24.9 months) in the chemotherapy group as compared with 2.3 months (range: $0.1-31.8$ months) in the supportive care group (Fig. 1a). In univariate analysis, we found that the older patients aged $\geq 80$ years with poor ECOG per- formance (EOCG $=2$ ), high CCI $(\geq 2)$, and those who were undergoing supportive care were associated with poor OS (Table 3 and Fig. 1b-d, NsxnsXNKlxn). Additionally, in multivariate analysis, poor performance $(\mathrm{EOCG}=2)$, high CCI $(\geq 2)$, and supportive care were associated with poor OS. In the chemotherapy group, 
Table 4. Adverse effects of chemotherapy in elderly patients with pancreatic cancer

\begin{tabular}{lll}
\hline & \multicolumn{2}{l}{ Patients $(N=36)$} \\
\cline { 2 - 3 } & CTCAE: $1-2$ & CTCAE: $\geq 3$ \\
\hline General weakness & 8 & 0 \\
Nausea & 3 & 0 \\
Peripheral neuropathy & 3 & 2 \\
Anemia & 5 & 1 \\
Neutropenia & 4 & 2 \\
Pneumonitis & 2 & 1 \\
\hline
\end{tabular}

$50 \%(n=18)$ of patients received doublet chemotherapy. The regimen included chemotherapeutic drugs, such as gemcitabine/Abraxane and FOLFIRINOX. The regimens were used as follows: gemcitabine/Abraxane, $n=12$; FOLFIRINOX, $n=3$; gemcitabine/erlotinib, $n=3$; and gemcitabine single agent, $n=18$. Among these patients, 8 of 36 reduced dosage of chemotherapy ( 3 of 3 in FOLFIRINOX reduced $25 \%$ dose reduction of original dosage and 8 of 12 in gemcitabine/Abraxane reduced 25\% dose reduction of original dosage). Nine patients (30\%) received second-line chemotherapy. In the supportive care group, $55.8 \%(n=74)$ of patients survived $<3$ months. However, except for earlier deaths ( $<3$ months), the median survival obtained was 6.5 months in the supportive group. Table 4 showed adverse events of chemotherapy. In patients who received chemotherapy, most adverse effects were mild and manageable. The adverse effects over grade 3 included neutropenia, anemia, peripheral neuropathy, and pneumonitis (16.7\%).

\section{Discussion}

This study focused on the treatment patterns and their outcomes in older patients with metastatic pancreatic cancer. Only $10.7 \%$ of the total number of elderly patients with metastatic pancreatic cancer received gemcitabinebased doublet chemotherapy; however, their OS was comparable to younger patients with metastatic pancreatic cancer.

Based on our results, it can be seen that in the real world, only a few older patients $(36 / 167,21.6 \%)$ with metastatic pancreatic cancer receive chemotherapy. Nonetheless, in these few patients, chemotherapy increased the survival rate, with the combination chemotherapy showing the survival benefit similar to younger patients. One

Treatment for Elderly Patients with

Metastatic Pancreatic Cancer reason for this is that there is no adequate guide and specific indication on who will benefit from chemotherapy in these older patients. An assessment of medical comorbidities and functional status plays a key role in determining fitness for undergoing intensive chemotherapeutic regimens in this important subset of patients. This study clarified the indications for chemotherapy that was vaguely determined by each physician in the clinic during the treatment of older patients with pancreatic cancer. As shown in this study, good performance status and the low CCI were good indices during the selection of appropriate candidates for active chemotherapy in older patients with metastatic pancreatic cancer. Most of these patients in the chemotherapy group tolerated the therapy well and showed a good response to gemcitabine-containing doublet chemotherapy. Possible strategies to improve tolerability without decreasing the efficacy of chemotherapy include dose reduction, schedule modification, and growth factor support.

Although low clinical trial enrollment is typical with older patients and insufficient data exist, most of the pancreatic cancer patients we consult in clinical practice are older. In particular, pancreatic cancer is a very aggressive tumor, and for this reason, older patients with this condition have been a difficult subsegment of population to manage, regarding strategic planning of drug selection for aggressive chemotherapy. However, in recent years, many new drugs have been developed, including immune checkpoint inhibitors and therapeutic vaccines, and these medications are evolving in terms of their effects and side effects; hence, older patients should not be overlooked in such treatment opportunities. Most new drug studies do not only limit patient's age but also differ in the distribution of patients enrolled in a new drug study, compared with those monitored in clinical trials, making it difficult for clinicians to apply the trial results only in clinical practice.

Thirty-seven percent of good performance and low CCI decline chemotherapy due to concern of the side effect of chemotherapy. Despite our study findings that most of the older patients with pancreatic cancer who may do well with chemotherapy are often denied such treatment, this study does not preclude limitations. First, because the study was of a retrospective design, we had limited control over the data collection and had to rely on accurate recordkeeping by other staff for our analyses. Second, due to our small-scale study population size, there could be higher data variability. We hope that in the future, drug researchers would consider older patients for the treatment category.

Chemotherapy 2021;66:107-112 111 


\section{Conclusions}

Our findings in this study supported that aggressive chemotherapy for older pancreatic cancer patients (with good performance and low CCI) might have tolerable side effects and a favorable outcome to improve their survival.

\section{Statement of Ethics}

This study was approved by the Institutional Review Board with a waiver for informed consent, owing to its retrospective design.

\section{Conflict of Interest Statement}

The authors declare no conflicts of interest.

\section{Funding Sources}

None of the authors have financial disclosures or personal relationships with other people or organizations that could inappropriately influence this work. There was no funding for this study.

\section{Author Contributions}

Hyun Ae Jung and Joo Young Jung designed this study. Hyun Ae Jung, Bo Ram Han, Ho Young Kim, Hyo Jung Kim, and Dae Young Zang gathered patients' data and analyzed the data. Hyun Ae Jung drafted the manuscript. All the authors reviewed the manuscript and made suggestions to finalize the document.

\section{References}

1 Parry C, Kent EE, Mariotto AB, Alfano CM, Rowland JH. Cancer survivors: a booming population. Cancer Epidemiol Biomarkers Prev. 2011;20(10):1996-2005.

2 Li D, Capanu M, Yu KH, Lowery MA, Kelsen DP, O'Reilly EM. Treatment, outcomes, and clinical trial participation in elderly patients with metastatic pancreas adenocarcinoma. Clin Colorectal Cancer. 2015;14(4):269-e1.

3 Burris HA 3rd, Moore MJ, Andersen J, Green MR, Rothenberg ML, Modiano MR, et al. Improvements in survival and clinical benefit with gemcitabine as first-line therapy for patients with advanced pancreas cancer: a randomized trial. J Clin Oncol. 1997;15(6):240313.

4 Oberstein PE, Hershman DL, Khanna LG, Chabot JA, Insel BJ, Neugut AI. Uptake and patterns of use of gemcitabine for metastatic pancreatic cancer: a population-based study. Cancer Invest. 2013;31(5):316-22.

5 Von Hoff DD, Ervin T, Arena FP, Chiorean EG, Infante J, Moore M, et al. Increased survival in pancreatic cancer with nab-paclitaxel plus gemcitabine. N Engl J Med. 2013;369(18): 1691-703.

6 Conroy T, Desseigne F, Ychou M, Bouché O, Guimbaud R, Bécouarn Y, et al. Folfirinox versus gemcitabine for metastatic pancreatic cancer. N Engl J Med. 2011;364(19):1817-25.

7 Cunningham D, Chau I, Stocken DD, Valle JW, Smith D, Steward W, et al. Phase iii randomized comparison of gemcitabine versus gemcitabine plus capecitabine in patients with advanced pancreatic cancer. J Clin Oncol. 2009;27(33):5513-8.
8 Oettle H, Richards D, Ramanathan RK, van Laethem JL, Peeters M, Fuchs M, et al. A phase iii trial of pemetrexed plus gemcitabine versus gemcitabine in patients with unresectable or metastatic pancreatic cancer. Ann Oncol. 2005;16(10): 1639-45.

9 Stathopoulos GP, Syrigos K, Aravantinos G, Polyzos A, Papakotoulas P, Fountzilas G, et al. A multicenter phase iii trial comparing irinotecan-gemcitabine (IG) with gemcitabine $(\mathrm{G})$ monotherapy as first-line treatment in patients with locally advanced or metastatic pancreatic cancer. Br J Cancer. 2006;95(5):587-92.

10 Charlson ME, Pompei P, Ales KL, MacKenzie CR. A new method of classifying prognostic comorbidity in longitudinal studies: development and validation. J Chronic Dis. 1987; 40(5):373-83. 